\title{
Semantics of Propositional Fuzzy Modal Logic with Evaluated Syntax and its Application to Fuzzy Decision Implications
}

\author{
Xiaodong Pan ${ }^{1}$, Yang $\mathrm{Xu}^{2}$ \\ ${ }^{1,2}$ School of Mathematics, Southwest Jiaotong University, \\ West Section, High-tech Zone, \\ Chengdu, Sichuan, 611756, P.R. China \\ ${ }^{1}$ E-mail:xdpan1@163.com \\ ${ }^{2}$ E-mail:xuyang@swjtu.edu.cn
}

Received 10 February 2015

Accepted 27 October 2015

\begin{abstract}
This paper deals with propositional fuzzy modal logic with evaluated syntax based on MV-algebras. We focus on its semantical theory from the viewpoint of Pavelka's graded semantics of propositional fuzzy logic, investigate the L-tautologies based on different Kripke frames. We also define the notion of Lsemantical consequence operation, its some basic properties are obtained. Finally, this paper considers the fuzzy decision implications in propositional fuzzy modal logic with evaluated syntax based on MValgebras, and presents a kind of semantical characteristics of fuzzy decision implications. Moreover, we introduce the notions of possible and necessary fuzzy decision implication, and their semantical characteristics are presented as well.
\end{abstract}

Keywords: Propositional fuzzy modal logic; Evaluated syntax; L-tautology; MV-algebra; Consequence operation; Fuzzy decision implication.

\section{Introduction}

Fuzzy logics with evaluated syntax was introduced by Pavelka ${ }^{18}$ in 1979 . Pavelka broke through the traditional method of fuzzy logics, and established a kind of graded and complete fuzzy propositional logic. In this kind of fuzzy logics, not only the set of truth values is expanded, but also the formal language are extended by a set of logical (truth) constants that are names of all truth values, and some formulas which are not always fully true are introduced into the set of axioms, each formula being in the syntax assigned a value. The inference rules can transmit the truth values among formulas. In other words, the truth values of formulas as conclusion can be obtained from the truth values of formulas as premise by computation. After that, Novák ${ }^{15-17}$ extended Pavelka's fuzzy logic to firstorder fuzzy logic. Since the process of inference involves the transmission of truth values of formulas, and so fuzzy logic with evaluated syntax is suitable to model human's reasoning based on fuzzy information, thus making it possible to build artificially computer-based systems able to simulate human's intelligence by computer.

One of the most important directions extending fuzzy logic is the modal extensions of fuzzy logics, there are already various modal extensions of fuzzy logics, most of which are based on finitely valued logics with traditional syntax, see Ref. 10-13. The 
basic idea was to retain the general notion of possible world semantics, while allowing formulas to have values in a many-valued space, at each possible world. What seems not to have been considered is allowing the accessibility relation between possible worlds itself to be many-valued. But many-valued accessibility is a very natural notion. After all, some worlds alternative to this one are more relevant, others less, as one intuitively thinks of these things. In Ref. 6, 7, Bou also developed fuzzy modal logic with many-valued accessibility relation in the traditional way.

In formal concept analysis (FCA), one important aspect is the description of attribute dependencies in symbolic data. One of the most important means is to describe the dependencies by rules $A \Rightarrow B$ meaning that one can derive $B$ from $A$, which are called attribute implications and are interpreted in tables describing objects and attributes. Entries in a table say whether or not a particular object has a particular attribute. Then, $A \Rightarrow B$ is true in a table if each object having all attributes from $A$ has also all attributes from $B$. An implications may be valid in a set of data, if no data would violate the implication.

In Ref. 19, Pollandt introduced the notion of fuzzy implication, which extends implications in fuzzy cases. Afterwards Bělohlávek ${ }^{1-5}$ studied several aspects of fuzzy implications from the point of view of fuzzy logic, he presented the semantical and syntactical characteristics of fuzzy implications in two style, i.e., in crisp style and in Pavelka-style, and in two settings, i.e., within a data table with fuzzy attributes and in the logic way. As an extension of implication, decision implication was introduced by Qu et. al. in Ref. 20. Compared with implications, decision implication is constructed on two sets of attributes, called condition attributes and decision attributes, instead of only one set of attributes. In Ref. 24 , following Bělohlávek's work ${ }^{2-4}$, Zhai et.al. extended decision implication to the fuzzy setting and provides the semantical and syntactical characteristics of fuzzy decision implications.

It is well known that reasoning about fuzzy decision implications plays a crucial role in several areas of computer science and data engineering in particular. Two most important areas are databases and data mining. In the former, the rule $A \Rightarrow B$ are called functional dependency, they are used for management of data redundancy and for database design; in the latter, these rules are widely used to represent knowledge.

In this paper, we establish a kind of graded semantic theory for propositional fuzzy modal logic with evaluated syntax based on MV-algebras, allow the accessibility relation between worlds also to be many-valued, and introduce graded modal connectives into the formal languages. We also propose the notion of satisfiability with certain level for a set of formulas from the viewpoint of approximating reasoning. Furthermore, we define the semantical consequence operation with respect to a set of formulas, and the consistency of fuzzy information. Finally, following the work of Zhai ${ }^{24}$, this paper considers fuzzy decision implications in propositional fuzzy modal logic with evaluated syntax based on MV-algebras, introduces the notions of possible and necessary fuzzy decision implication, and provides the semantical characteristics of these fuzzy decision implications. Some basic results are obtained.

\section{Preliminaries}

The set of truth values is supposed to form an MValgebra $^{8}$. An algebraic structure $\mathscr{L}=\left(L, \oplus,{ }^{\prime}, 0\right)$ with similarity type $\langle 2,1,0\rangle$ is an MV-algebra if and only if $(L, \oplus, 0)$ is an abelian monoid with neutral element 0 , and if furthermore for all $x, y \in L$ there hold true

(i) $x^{\prime \prime}=x$,

(ii) $x \oplus 0^{\prime}=0^{\prime}$,

(iii) $\left(x^{\prime} \oplus y\right)^{\prime} \oplus y=\left(y^{\prime} \oplus x\right)^{\prime} \oplus x$.

Such an MV-algebra is nontrivial if and only if it contains at least two elements. By denoting $x \otimes y=$ $\left(x^{\prime} \oplus y^{\prime}\right)^{\prime}$ and $1=0^{\prime}$, it follows that $\left(L, \otimes,^{\prime}, 1\right)$ is also an MV-algebra, which is known as the dual MValgebra of $\left(L, \oplus,{ }^{\prime}, 0\right)$. Notice also that it is possible to derive a lattice structure from that of MV-algebra, since $x \leqslant_{L} y$ iff $x^{\prime} \oplus y=1$ iff $x \otimes y^{\prime}=0$ defines a partial order on $L$, in such a way that $\left(L, \leqslant_{L}, \vee, \wedge, 0,1\right)$ is a lattice with the meet and join operations respectively given, for all $x, y \in L$, by $x \wedge y=\left(x^{\prime} \otimes y\right)^{\prime} \otimes y$, $x \vee y=\left(x^{\prime} \oplus y\right)^{\prime} \oplus y$, and 0 and 1 are the bottom 
and top elements, respectively. The following is an equivalent definition of MV-algebra, which is also known as a lattice implication algebra which has been introduced by Xu et. al. in Ref. 22, 23. The equivalency between these two definitions has been proved by Wang in Ref. 21.

Definition 1. A bounded lattice $(L, \vee, \wedge, 0,1)$ with order reversing involution ' and a binary operation $\rightarrow$ is called a lattice implication algebra if it satisfies the following axioms: for all $x, y, z \in L$,

$\left(I_{1}\right) x \rightarrow(y \rightarrow z)=y \rightarrow(x \rightarrow z)$,

(I $\left.I_{2}\right) x \rightarrow x=1$,

(I3) $x \rightarrow y=y^{\prime} \rightarrow x^{\prime}$,

(I $\left.I_{4}\right) x \rightarrow y=y \rightarrow x=1 \Rightarrow x=y$,

(I5) $(x \rightarrow y) \rightarrow y=(y \rightarrow x) \rightarrow x$,

$\left(L_{1}\right)(x \vee y) \rightarrow z=(x \rightarrow z) \wedge(y \rightarrow z)$,

$\left(L_{2}\right)(x \wedge y) \rightarrow z=(x \rightarrow z) \vee(y \rightarrow z)$.

In what follows, we will use the equivalent definition in most cases for convenience.

Let $\mathscr{P}_{F M}$ be the propositional fuzzy modal logic system with evaluated syntax based on MValgebras, the language $J$ of $\mathscr{P}_{F M}$ consists of: (1) the set of propositional variable: $S=\left\{p_{1}, p_{2}, p_{3}, \cdots\right\}$; (2) the set of logical constants: $\bar{L}=\{\bar{a} \mid a \in L\}$; (3) logical connectives: $\neg, \Rightarrow,\left\{\square_{\beta}\right\}_{\beta \in L \backslash\{0\}}$, where for any $\beta \in L \backslash\{0\}, \square_{\beta}$ (it is necessary to the degree $\beta$, also read "box beta") is modal connective; (4) auxiliary symbols: $),($.

The set $\mathscr{F}_{J}$ of well-formed formulas of $\mathscr{P}_{F M}$ is the least set $Y$ satisfying the following conditions: (1) $S \subset Y$; (2) $\bar{L} \subset Y$; (3) if $A, B \in Y$, then $\neg A, A \Rightarrow B \in Y$, and for any $\beta \in L \backslash\{0\}, \square_{\beta} A \in Y$.

Remark 1. From the viewpoint of universal algebra, $\mathscr{F}_{J}$ is the free algebra on $S$ with respect to the type $T=\bar{L} \cup\left\{\neg, \Rightarrow,\left\{\square_{\beta}: \beta \in L \backslash\{0\}\right\}\right\}$, where $\bar{a} \in \bar{L}$ is a 0 -ary operation.

In what follows, unless otherwise stated, $L$ always represents a complete MV-algebra (For more details, please refer to Ref. 9), and the set $\mathbb{N} \backslash\{0\}$ will be denoted by $\mathbb{N}^{+}$.

For the sake of convenience, we introduce some abbreviations as follows: for any $A, B, C \in \mathscr{F}_{J}, n \in$ $\mathbb{N}^{+}, \beta \in L \backslash\{0\}$,

$A \vee B \triangleq(A \Rightarrow B) \Rightarrow B, A \wedge B \triangleq \neg(\neg A \vee \neg B), A \& B \triangleq$ $\neg(A \Rightarrow \neg B), A \nabla B \triangleq \neg A \Rightarrow B, A \Leftrightarrow B \triangleq(A \Rightarrow$
$B) \wedge(B \Rightarrow A), \diamond_{\beta} A \triangleq \neg \square_{\beta} \neg A, A^{0} \triangleq \overline{1}, A^{n} \triangleq$ $A^{n-1} \& A, 0 A \triangleq \overline{0}, n A \triangleq(n-1) A \nabla A$.

By $L^{\mathscr{F}_{J}}$ we denote the set of all L-fuzzy sets on $\mathscr{F}_{J}$, that is, the set of all mappings from $\mathscr{F}_{J}$ to $L$, and call an element of $L^{\mathscr{F _ { J }}}$ fuzzy information, and denote the greatest L-fuzzy set on $\mathscr{F}_{J}$ by $1_{\mathscr{F}_{J}}$, that is to say, an L-fuzzy set which assigns each element of $\mathscr{F}_{J}$ the greatest element 1 . Let $Y \in L^{\mathscr{\mathscr { F } _ { J }}}$, if $\operatorname{supp} Y=\left\{A \in \mathscr{F}_{J} \mid Y(A)>0\right\}$ is a finite subset of $\mathscr{F}_{J}$. Then $Y$ is called a finite L-fuzzy set, or a finite set for short.

In a complete MV-algebra $L$, define a unary operator $*: L \rightarrow L$ for truth-stressing hedge (hedge for short, reads "very true"), satisfies: $1^{*}=1, a^{*} \leqslant a$, $(a \rightarrow b)^{*} \leqslant a^{*} \rightarrow b^{*}$ and $a^{* *}=a$ for all $a, b \in L$. Two boundary cases of hedges are (i) identity, i.e. $a^{*}=a(a \in L)$; (ii) globalization

$$
a^{*}= \begin{cases}1, & \text { if } a=1 \\ 0, & \text { otherwise } .\end{cases}
$$

Let $U$ be the universe and $A, B \in L^{U}$. We define a subsethood degree $S(A, B)=\bigwedge_{u \in U}(A(u) \rightarrow B(u))$, which generalizes the classical subsethood relation $\subseteq$.

In the fuzzy logics with evaluated syntax, the most elementary concept is evaluated formula.

Definition 2. ${ }^{14}$ An evaluated formula is a couple $a / A$, where $A \in \mathscr{F}_{J}$ is a formula and $a \in L$ is its syntactic evaluation.

Inference rules in the fuzzy logics with evaluated syntax deal with the evaluated formulas.

Definition 3. ${ }^{14}$ An $n$-ary inference rule $r$ in the fuzzy logics with evaluated syntax is a scheme

$$
r: \frac{a_{1} / A_{1}, \cdots, a_{n} / A_{n}}{r^{e v l}\left(a_{1}, \cdots, a_{n}\right) / r^{\text {syn }}\left(A_{1}, \cdots, A_{n}\right)},
$$

using which the evaluated formulas $a_{1} / A_{1}, \cdots, a_{n} / A_{n}$ are assigned the evaluated formula $r^{\text {syn }}\left(A_{1}, \cdots, A_{n}\right)$. The syntactic operation $r^{\text {syn }}$ is a partial $n$-ary operation on $\mathscr{F}_{J}$ and the evaluated operation $r^{e v l}$ is an $n$-ary lower semicontinuous operation on $L$.

Based on the above evaluated formula and inference rule, the concept of evaluated formal proof can be introduced. 
Definition 4. ${ }^{14}$ An evaluated formal proof of a formula $A$ from the fuzzy set $X \in L^{\mathscr{F}_{J}}$ is a finite sequence of evaluated formulas

$$
\omega:=a_{0} / A_{0}, a_{1} / A_{1}, \cdots, a_{n} / A_{n}
$$

such that $A_{n}:=A$ and for each $i \leqslant n$, either there exists an $n$-ary inference rule $r$ such that $a_{i} / A_{i}:=$ $r^{e v l}\left(a_{i_{1}}, \cdots, a_{i_{n}}\right) / r^{\text {syn }}\left(A_{i_{1}}, \cdots, A_{i_{n}}\right), i_{1}, \cdots, i_{n}<i$, or $a_{i} / A_{i}:=X\left(A_{i}\right) / A_{i}$.

The evaluation $a_{n}$ of the last member in the above formal proof $\omega$ is the value of $\omega$, usually denoted by $\operatorname{Val}(\omega)$.

\section{L-tautology theory}

In this section, we investigate L-tautologies in $\mathscr{P}_{F M}$. Semantically, the propositional fuzzy logic based on MV-algebras can be characterised by a structure $S_{\mathscr{P}_{F}}=\left\langle L, D,\left\{f_{c} ; c \in\{\neg, \Rightarrow\}\right\}\right\rangle$, where $L$ is an MValgebra, $D \subset L \backslash\{0\}$ is the set of designated values which are the values that are preserved in valid inferences. For each connective, $c, f_{c}$ is the truth function it denotes.

An L-valued Kripke frame is a pair $\mathscr{F}=\langle W, R\rangle$ where $W$ is a non-empty set of worlds, $R$ is a binary L-fuzzy accessibility relation on $W$. An interpretation for $\mathscr{P}_{F M}$ is a structure (or model) $\left\langle W, R, S_{\mathscr{P}_{F}}, v\right\rangle$ (denoted as $\langle W, R, v\rangle$ below when without confusion or errors), where $\langle W, R\rangle$ is an L-valued Kripke frame, $S_{\mathscr{P}_{F}}$ is the structure as defined above, and for each propositional parameter, $p$, and world, $\omega$, $v$ assigns the parameter a value, $v_{\omega}(p)$, in $L$. This is extended to a map from $\mathscr{F}_{J}$ to $L$ at a world $\omega$ by applying the following truth functions recursively:

- $v_{\omega}(\neg A)=\left(v_{\omega}(A)\right)^{\prime}$;

- $v_{\omega}(A \Rightarrow B)=v_{\omega}(A) \rightarrow v_{\omega}(B)$;

- for all $a \in L, v_{\omega}(\bar{a})=a$;

- for any $\beta \in L \backslash\{0\}, v_{\omega}\left(\square_{\beta} A\right)=\bigwedge\left\{v_{\omega^{\prime}}(A): \omega^{\prime} \in\right.$ $\left.W, R\left(\omega, \omega^{\prime}\right) \geqslant \beta\right\}$

- for any $\beta \in L \backslash\{0\}, v_{\omega}\left(\diamond_{\beta} A\right)=\bigvee\left\{v_{\omega^{\prime}}(A): \omega^{\prime} \in\right.$ $\left.W, R\left(\omega, \omega^{\prime}\right) \geqslant \beta\right\}$.

Definition 5. Let $0<\alpha \in L$, a modal formula $A$ is $\alpha$-tautology in a structure $\mathscr{M}=\langle W, R, v\rangle$, denoted
$\mathscr{M} \models{ }_{\alpha} A$, provided $\bigwedge_{\omega \in W} v_{\omega}(A)=\alpha$. A modal formula $A$ is $\alpha$-tautology in a frame $\mathscr{F}=\langle W, R\rangle$, denoted $\mathscr{F} \models_{\alpha} A$, provided that $A$ is an $\alpha$-tautology in any structure based on $\mathscr{F}$. And if $\mathbf{K}$ is a class of frames then we write $\mathbf{K} \models_{\alpha} A$ to mean that $A$ is an $\alpha$-tautology in all frames in this class.

For any kind of $\alpha$-tautology $A$ mentioned above, if there exists $\omega \in W$ in some structure $\mathscr{M}$ such that $v_{\omega}(A)=\alpha$, then $A$ is called a reachable $\alpha$ tautology.

The set of all $\alpha$-tautologies in a structure $\mathscr{M}$ (a frame $\mathscr{F}$, a class of frames $\mathbf{K}$ ) will be denoted by $\alpha-\operatorname{Tau}(\mathscr{M})(\alpha-\operatorname{Tau}(\mathscr{F}), \alpha-\operatorname{Tau}(\mathbf{K}))$. The set of all reachable $\alpha$-tautologies in a structure $\mathscr{M}$ (a frame $\mathscr{F}$, a class of frames $\mathbf{K}$ ) will be denoted by $[\alpha)$ $\operatorname{Tau}(\mathscr{M})([\alpha)-\operatorname{Tau}(\mathscr{F}),[\alpha)-\operatorname{Tau}(\mathbf{K}))$.

In the following, we always assume that $\alpha>0$ unless otherwise stated.

Example 1. For any $\beta \in L \backslash\{0\}$, the following modal formulas are 1-tautology in all frames:

(1) $\square_{\beta}(A \wedge B) \Leftrightarrow\left(\square_{\beta} A \wedge \square_{\beta} B\right)$,

$\diamond_{\beta}(A \vee B) \Leftrightarrow\left(\diamond_{\beta} A \vee \diamond_{\beta} B\right)$

(2) for all $\bar{a} \in \bar{L}, \square_{\beta}(\bar{a} \Rightarrow A) \Leftrightarrow\left(\bar{a} \Rightarrow \square_{\beta} A\right)$;

(3) $\square{\beta_{i}}_{i} A \Rightarrow \square_{\beta_{j}} A, \diamond_{\beta_{j}} A \Rightarrow \diamond_{\beta_{i}} A$, if $\beta_{i} \leqslant \beta_{j}$;

(4) $\neg \neg \square_{\beta} A \Leftrightarrow \square_{\beta} \neg \neg A$;

(5) all tautologies in propositional fuzzy logic.

Theorem 1. If $L$ is a completely distributive lattice, then for any $\beta \in L \backslash\{0\}, \square_{\beta}(A \Rightarrow B) \Rightarrow\left(\square_{\beta} A \Rightarrow\right.$ $\left.\square_{\beta} B\right)$ is 1-tautology in all frames.

Proof. It suffices to show that in any structure $\mathscr{M}=\langle W, R, v\rangle$ and any world $\omega \in W, v_{\omega}\left(\square_{\beta}(A \Rightarrow\right.$ $B)) \leqslant v_{\omega}\left(\square_{\beta} A \Rightarrow \square_{\beta} B\right)$, that is, $\bigwedge\left\{v_{\omega^{\prime}}(A) \rightarrow\right.$ $\left.v_{\omega^{\prime}}(B): R\left(\omega, \omega^{\prime}\right) \geqslant \beta, \omega^{\prime} \in W\right\} \leqslant \bigwedge\left\{v_{\omega^{\prime}}(A):\right.$ $\left.R\left(\omega, \omega^{\prime}\right) \geqslant \beta, \omega^{\prime} \in W\right\} \rightarrow \bigwedge\left\{v_{\omega^{\prime}}(B): R\left(\omega, \omega^{\prime}\right) \geqslant\right.$ $\left.\beta, \omega^{\prime} \in W\right\}$. Let $X=\left\{\omega^{\prime}: R\left(\omega, \omega^{\prime}\right) \geqslant \beta, \omega^{\prime} \in W\right\}$, and let $a_{x}$ and $b_{x}$ be $v_{x}(A)$ and $v_{x}(B)$, respectively. We will show that: $\bigwedge\left\{a_{x} \rightarrow b_{x}: x \in X\right\} \leqslant \bigwedge\left\{a_{x}: x \in\right.$ $X\} \rightarrow \bigwedge\left\{b_{x}: x \in X\right\}$.

Since for any $x_{0} \in X, \wedge\left\{a_{x} \rightarrow b_{x}: x \in X\right\} \leqslant$ $a_{x_{0}} \rightarrow b_{x_{0}} \leqslant \bigwedge\left\{a_{x}: x \in X\right\} \rightarrow b_{x_{0}}$; note that $L$ is completely distributive, it follows from the arbitrariness of $x_{0}$ that $\bigwedge\left\{a_{x} \rightarrow b_{x}: x \in X\right\} \leqslant \bigwedge_{x \in X}\left\{\wedge\left\{a_{x}: x \in\right.\right.$ $\left.X\} \rightarrow b_{x}\right\}=\bigwedge\left\{a_{x}: x \in X\right\} \rightarrow \bigwedge\left\{b_{x}: x \in X\right\}$. This completes the proof. 
Proposition 2. For any $\beta \in L \backslash\{0\}$, if $A \in$ 1-Tau( $\mathscr{M})(1-\operatorname{Tau}(\mathscr{F}), 1-\operatorname{Tau}(\mathbf{K}))$, than $\square_{\beta} A \in 1$ $\operatorname{Tau}(\mathscr{M})(1-\operatorname{Tau}(\mathscr{F}), 1-\operatorname{Tau}(\mathbf{K}))$.

Proof. Suppose that $\square_{\beta} A \notin 1-\operatorname{Tau}(\mathscr{M})$. Then there is some world in the structure $\mathscr{M}=\langle W, R, v\rangle$, $\omega$, such that $v_{\omega}\left(\square_{\beta} A\right) \neq 1$. Thus, for some world $\omega^{\prime} \in W$ such that $R\left(\omega, \omega^{\prime}\right) \geqslant \beta, v_{\omega^{\prime}}(A) \neq 1$. Hence $A \notin 1-\operatorname{Tau}(\mathscr{M})$. The rest are obvious.

Proposition 3. Let $\mathscr{M}=\langle W, R, v\rangle$ be a structure and $\beta \in L \backslash\{0\}$. If $R$ is $\beta$-reflexive and $A \in[\alpha)$ $\operatorname{Tau}(\mathscr{M})$, than $\square_{\beta} A \in[\alpha)-\operatorname{Tau}(\mathscr{M})$.

Proof. Since $A \in[\alpha)-\operatorname{Tau}(\mathscr{M})$, then $\bigwedge_{\omega \in W} v_{\omega}(A)=\alpha$ and there is some world $\omega_{0} \in W$ such that $v_{\omega_{0}}(A)=\alpha$. Note that $R$ is $\beta$-reflexive, that is, $R(\omega, \omega) \geqslant \beta$ for any $\omega \in W$. Thus,

$\bigwedge_{\omega \in W} v_{\omega}\left(\square_{\beta} A\right)=\bigwedge_{\omega \in W}\left(\bigwedge\left\{v_{\omega^{\prime}}(A): R\left(\omega, \omega^{\prime}\right) \geqslant\right.\right.$ $\beta\})=\bigwedge_{\omega \in W, \omega \neq \omega_{0}}\left(\bigwedge\left\{v_{\omega^{\prime}}(A): R\left(\omega, \omega^{\prime}\right) \geqslant \beta\right\}\right) \wedge$ $\bigwedge\left\{v_{\omega^{\prime}}(A): R\left(\omega_{0}, \omega^{\prime}\right) \geqslant \beta\right\}=\alpha$.

Hence, $\square_{\beta} A \in[\alpha)-\operatorname{Tau}(\mathscr{M})$.

The following corollaries are straightforward.

Corollary 4. Let $\mathscr{F}=\langle W, R\rangle$ be a frame and $\beta \in L \backslash\{0\}$. For any structure $\mathscr{M}=\langle W, R, v\rangle$, if $R$ is $\beta$-reflexive and $A \in[\alpha)$-Tau( $\mathscr{F})$, than $\square_{\beta} A \in[\alpha)$ $\operatorname{Tau}(\mathscr{F})$.

Corollary 5. Let $\mathbf{K}$ be a class of frames and $\beta \in L \backslash\{0\}$. For any frame $\mathscr{F} \in \mathbf{K}$ and any structure $\mathscr{M}=\langle W, R, v\rangle$ based on $\mathscr{F}$, if $R$ is $\beta$-reflexive and $A \in[\alpha)-\operatorname{Tau}(\mathbf{K})$, than $\square_{\beta} A \in[\alpha)-\operatorname{Tau}(\mathbf{K})$.

Proposition 6. Let $\mathscr{M}=\langle W, R, v\rangle$ be a structure. For any $\beta \in L \backslash\{0\}$, the following conclusions hold: (1) if $R$ is $\beta$-reflexive, then $\square_{\beta} A \Rightarrow A \in 1-\operatorname{Tau}(\mathbf{M})$;

(2) if $R$ is symmetric, then $A \Rightarrow \square_{\beta} \diamond_{\beta} A \in 1$ $\operatorname{Tau}(\mathbf{M})$;

(3) if $R$ is max-min transitive, then $\square_{\beta} \Rightarrow \square_{\beta} \square_{\beta} A \in$ $1-\operatorname{Tau}(\mathbf{M})$.

Proof. For (1), since $R(\omega, \omega) \geqslant \beta$ for any $\omega \in W$, so $v_{\omega}\left(\square_{\beta} A\right)=\bigwedge\left\{v_{\omega^{\prime}}: R\left(\omega, \omega^{\prime}\right) \geqslant \beta\right\} \leqslant v_{\omega}(A)$, as required.

For (2), since $R\left(\omega_{1}, \omega_{2}\right)=R\left(\omega_{2}, \omega_{1}\right)$ for any $\omega_{1}, \omega_{2} \in W$, thus for any $\omega \in W$, if $R\left(\omega, \omega^{\prime}\right) \geqslant$ $\beta$, then $v_{\omega}(A) \leqslant \bigvee_{R\left(\omega^{\prime}, \omega^{\prime \prime}\right) \geqslant \beta} v_{\omega^{\prime \prime}}(A)=v_{\omega^{\prime}}\left(\diamond_{\beta} A\right)$. So $v_{\omega}(A) \leqslant \bigwedge_{R\left(\omega, \omega^{\prime}\right) \geqslant \beta} v_{\omega^{\prime}}\left(\diamond_{\beta} A\right)$, that is, $v_{\omega}(A) \leqslant$ $v_{\omega}\left(\square_{\beta} \diamond_{\beta} A\right)$, as required.
For (3), we will show that $v_{\omega}\left(\square_{\beta} A\right) \leqslant$ $v_{\omega}\left(\square_{\beta} \square_{\beta} A\right)$ for any $\omega \in W$. If $R\left(\omega, \omega^{\prime}\right) \geqslant \beta$, then we can have $R\left(\omega, \omega^{\prime \prime}\right) \geqslant \beta$ from $R\left(\omega^{\prime}, \omega^{\prime \prime}\right) \geqslant \beta$ and the transitivity of $R$. So $\left\{v_{\omega^{\prime \prime}}(A): R\left(\omega^{\prime}, \omega^{\prime \prime}\right) \geqslant\right.$ $\beta\} \subseteq\left\{v_{\omega^{\prime \prime}}(A): R\left(\omega, \omega^{\prime \prime}\right) \geqslant \beta\right.$. Thus, $\wedge\left\{v_{\omega^{\prime \prime}}(A):\right.$ $\left.R\left(\omega, \omega^{\prime \prime}\right) \geqslant \beta\right\} \leqslant \bigwedge\left\{v_{\omega^{\prime \prime}}(A): R\left(\omega^{\prime}, \omega^{\prime \prime}\right) \geqslant \beta\right\}$. So $\bigwedge\left\{v_{\omega^{\prime \prime}}(A): R\left(\omega, \omega^{\prime \prime}\right) \geqslant \beta\right\} \leqslant \bigwedge\left\{\bigwedge\left\{v_{\omega^{\prime \prime}}(A)\right.\right.$ : $\left.\left.R\left(\omega^{\prime}, \omega^{\prime \prime}\right) \geqslant \beta\right\}: R\left(\omega, \omega^{\prime}\right) \geqslant \beta\right\}$. This completes the proof.

\section{Semantic consequence operation and consistency of information}

In 1979, Pavelka defined the semantic and syntactic consequence operations as self-mappings on $L^{\mathscr{F}_{J}}$, the semantic and syntactic deductions were presented in the form of L-consequence operation. In this way, we define the notion of L-consequence operation in $\mathscr{P}_{F M}$ in this section.

As a naturally extension of the notion of validity in propositional fuzzy logic with evaluated syntax, we understand validity in $\mathscr{P}_{F M}$ as follows:

Let $\Sigma$ be a subset of $\mathscr{F}_{J}$ and $A \in \mathscr{F}_{J} . \Sigma \vDash_{\alpha} A$ if and only if for every structure, $\langle W, R, v\rangle$, and for every $\omega \in W$, whenever $v_{\omega}(B) \in D$ for every $B \in \Sigma$, $v_{\omega}(A) \in D$. Where $D \subset L \backslash\{0\}$ is the set of designated truth values which satisfies: for any $\alpha_{1} \in D$, if $\alpha_{1} \leqslant \alpha_{2}$, then $\alpha_{2} \in D$.

Here it should be stressed that in classical sense, the corresponding set of designated truth values only contains one element 1, i.e., $D=\{1\}$. In Pavelka's and Novák's fuzzy logic with evaluated syntax, there is no explicit the set of designated truth values. In this paper, we generalizes the set $D$ of designated truth values into more general situation, here $D$ is only a subset of $L$ and an up-set. Further, you can restrict $D$ to a filter of $L$. We consider this due to the fact that usually people don't consider these propositions whose truth values are very small (close to 0 ) as the premises of an inference.

In the following, $D$ always denotes a set of designated values unless otherwise stated.

Definition 6. Let $M \subset \mathscr{F}_{J}$. Define $M_{D} \subset$ $L^{\mathscr{F}_{J}} \quad$ as $\quad M_{D} \triangleq\left\{X \in L^{\mathscr{F}_{J}} \mid \forall A \in \mathscr{F}_{J}\right.$, if $A \in$ $M$ and $A$ is not a constant, then $X(A) \in D$; if $A \in$ 
$M$ and $A=\bar{a}$ is a constant, then $X(A)=$ $a$; otherwise, $X(A)=0$. .

Let $X \in M_{D}$. For any $A \in M$, the value $X(A)$ represents the initial truth value of $A$ with regard to $M$, and $X$ is called information with regard to $M$ in $\mathscr{P}_{F M}$.

Definition 7. Let $\mathscr{M}=\langle W, R, v\rangle$ be a structure and $M \subset \mathscr{F}_{J}$. We say that $\mathscr{M}$ is a model of $M$, or $\mathscr{M}$ satisfies $M$ on the level of $D$ if there is a world $\omega$ such that $v_{\omega}(A) \in D$ for every $A \in M$ that is not a constant. We say that $M$ is satisfiable on the level of $D$ if there is a structure $\mathscr{M}=\langle W, R, v\rangle$ such that $\mathscr{M}$ is a model of $M$ on the level of $D$.

Proposition 7. $M$ is satisfiable on the level of $D$ if and only if there exist $X \in M_{D}$, structure $\mathscr{M}=$ $\langle W, R, v\rangle$ and world $\omega$ such that $v_{\omega} \geqslant X$.

Proof. From Definition 4.1 and 4.2, this is obvious.

Definition 8. Let $X \in M_{D}$. If $\bar{X} \in L^{\mathscr{F} J}$ satisfying

$$
\bar{X}(A)= \begin{cases}1, & \text { if there exists } B \in M \text { such that } \\ & X(B)=a>0 \text { and } A=\bar{a} \Rightarrow B ; \\ 0, & \text { otherwise. }\end{cases}
$$

then we say $\bar{X}$ a substitution of $X$ on the level of $D$.

If $\bar{X}$ is a substitution of $X$ on the level of $D$, then we can consider $\bar{X}$ as a classical subset of $\mathscr{F}_{J}$, and for every $A \in \mathscr{F}_{J}$, if $A \in M$, then $\bar{X}(\overline{X(A)} \Rightarrow A)=1$; otherwise, $\bar{X}(\overline{X(A)} \Rightarrow A)=0$.

Theorem 8. Let $\mathscr{M}=\langle W, R, v\rangle$ be a structure. Then $\mathscr{M}$ is a model of $M$ on the level of $D$ if and only if there is a world $\omega$ and $X \in M_{D}$, and a substitution $\bar{X}$ of $X$ on the level of $D$ such that $v_{\omega} \geqslant \bar{X}$.

Proof. By Proposition 4.1, here we only need to prove that for any world $\omega$ and $X \in M_{D}, v_{\omega} \geqslant X$ if and only if $v_{\omega} \geqslant \bar{X}$.

This is because that $v_{\omega} \geqslant \bar{X}$ if and only if for any $A \in \mathscr{F}_{J}$, if $A \in M$, then $v_{\omega}(\overline{X(A)} \Rightarrow A)=1$; if and only if for any $A \in \mathscr{F}_{J}$, if $A \in M$, then $v_{\omega}(\overline{X(A)}) \rightarrow$ $v_{\omega}(A)=1$; if and only if for any $A \in \mathscr{F}_{J}$, if $A \in M$, then $X(A) \leqslant v_{\omega}(A)$; if and only if $v_{\omega} \geqslant X$. This completes the proof.
Remark 2. One could see that by Theorem 4.1, the satisfiability of an L-fuzzy set of formulas can be convert into the satisfiability of a classical set of formulas.

In classical logic, we say that $A$ can be derived from $M$, that is to say, $M \vDash A$ if and only if for any valuation $v$, if $v(B)=1$ for any $B \in M$, then we have $v(A)=1$; we could show this deduction in the form of sets as well, $M \vDash A$ if and only if $A \in \bigcap\left\{T_{v} \mid M \subset T_{v}, v\right.$ is a valuation $\}$, where

$T_{v}=\{p \mid p$ is a well-formed formula and $v(p)=1\}$.

In the following, we generalize the notion of semantic deduction, and define the semantic deduction of $M$ on the level of $D$ in $\mathscr{P}_{F M}$ as follows:

Definition 9. Let $\mathscr{M}=\langle W, R, v\rangle$ be a structure and $M \subset \mathscr{F}_{J}$. Define the mapping: $\mathfrak{C}_{\mathscr{M}^{2} D}: M_{D} \rightarrow L^{\mathscr{F}_{J}}$ as follows: for any $X \in M_{D}$, and for any $A \in \mathscr{F}_{J}$, $\mathfrak{C}_{\mathscr{M}, D}^{M}(X)(A)=\bigwedge_{\omega \in W}\left\{v_{\omega}(A) \mid v_{\omega} \geqslant X\right\}$.

Remark 3. In definition 4.4, if $\left\{\omega \in W \mid v_{\omega} \geqslant X\right\}=$ $\emptyset$, i.e, there exists no $\omega \in W$ such that $v_{\omega} \geqslant X$, then we let $\mathfrak{C}_{\mathscr{M}, D}^{M}(X)=1_{\mathscr{F}_{J}}$, here we call $X$ inconsistent information in the structure $\mathscr{M}$. Conversely, if $\left\{\omega \in W \mid v_{\omega} \geqslant X\right\} \neq \emptyset$, then we call $X$ consistent information in the structure $\mathscr{M}$. If for any $X \in M_{D}, X$ is consistent in the structure $\mathscr{M}$, then $M$ is said to be consistent on the level of $D$ in the structure $\mathscr{M}$.

We just define these notions above in a structure $\mathscr{M}$, similarly, it is easy to extend them into a frame $\mathscr{F}=\langle W, R\rangle$, and a class of frames $\mathbf{K}$. Here we will not explain it again.

\section{The semantical characteristics of fuzzy decision implications}

As we mentioned previously, fuzzy decision implications play an important role in computer science and data engineering. In this section, let $U$ be the universe (the set of all attributes), $A \in L^{U}$ will be a formula in $\mathscr{P}_{F M}$. Let $T \in L^{U}$ (which can be also understood as a possible world), we can assign a value $\|A\|_{T}=S(A, T)$ to the formula $A$, which means the truth degree to which one believe that one object has the attribute $A$ in the environment $T$. 
Let $L^{C}, L^{D}$ be two systems of L-sets in the universes $C$ and $D$ respectively $(C$ and $D$ are two infinite sets of attributes, and satisfy $C \cap D=\emptyset)$. The notion of fuzzy decision implication introduced by Zhai in Ref. 24 is defined as follows:

Definition 10. A fuzzy decision implication is of the expression $A \Rightarrow B$, where $A \in L^{C}$ and $B \in L^{D}$. Here $A$ is the premise of the implication and $B$ the consequence of the implication.

It is worth noting that the study of fuzzy decision implication is not a special case of that of fuzzy implication, at least in syntactical aspect, that is, we cannot directly apply the deduction rules for fuzzy implications to fuzzy decision implications, since a lot of deduction rules are not valid in this case.

In Ref. 24, Zhai et. al. define several notions concerning the semantical aspect of fuzzy decision implications. Here, we define the true value of $A \Rightarrow B$ by altering slightly Zhai's definition.

Definition 11. For a fuzzy set $T \in L^{C \cup D}$, the degree to which $T$ respects $A \Rightarrow B$ is defined by

$$
\|A \Rightarrow B\|_{T}=S(A, T \cap C) \rightarrow S(A, T \cap D) .
$$

In Ref. 24, Zhai et. al. define $\|A \Rightarrow B\|_{T}$ as follow:

$$
\|A \Rightarrow B\|_{T}=S(A, T \cap C)^{*} \rightarrow S(A, T \cap D) .
$$

Where $*$ is a hedge. But neither Zhai consider $A \in$ $L^{C}$ (or $B \in L^{D}$ ) as a formula, nor define the notion of $\|A\|_{T}$. In fact, in Ref. 1-5, 20 and Ref. 24, fuzzy implications or fuzzy decision implications are only considered by taking as a whole in fuzzy attribute logic. It will be shown below, under our way, it is more natural to characterize the semantical and syntactical properties of fuzzy decision implications in $\mathscr{P}_{F M}$.

In the above definition, $\|A \Rightarrow B\|_{T}$ says that if $A$ is contained in $T \cap C$, then $B$ should be also contained in $T \cap D$. If $C=D$, then $\|A \Rightarrow B\|_{T}$ degenerates the corresponding notion with respect to fuzzy implication.

In the following, we introduce the notions of possible fuzzy decision implication and necessary fuzzy decision implication as follows:
Definition 12. Let $\beta \in L \backslash\{0\}$. A possible (necessary) fuzzy decision implication is of the expression $\diamond_{\beta}(A \Rightarrow B)\left(\square_{\beta}(A \Rightarrow B)\right)$, where $A \in L^{C}$ and $B \in L^{D}$ and $\diamond_{\beta}\left(\square_{\beta}\right)$ is the possible (necessary) modal operation, which means that it is possible (necessary) to the degree $\beta$. Here $A$ is the premise of the implication and $B$ the consequence of the implication.

Let $W$ be the collection of all L-fuzzy sets $T \in L^{U}$ and $R$ a binary L-fuzzy accessibility relation on $W$. Similarly, we can introduce the following definitions.

Definition 13. For a fuzzy set $T \in L^{C \cup D}$, the degree to which $T$ respects $\diamond_{\beta}(A \Rightarrow B)\left(\square_{\beta}(A \Rightarrow B)\right)$ is defined by $\left\|\diamond_{\beta}(A \Rightarrow B)\right\|_{T}=\bigvee\left\{\|A \Rightarrow B\|_{T}: T^{\prime} \in\right.$ $\left.L^{U}, R\left(T, T^{\prime}\right) \geqslant \beta\right\}$. $\left(\left\|\square_{\beta}(A \Rightarrow B)\right\|_{T}=\bigwedge\{\| A \Rightarrow\right.$ $\left.B \|_{T}: T^{\prime} \in L^{U}, R\left(T, T^{\prime}\right) \geqslant \beta\right\}$.)

The following theorem says that any fuzzy decision implications can be represented by the fuzzy implications which are fully true.

Theorem 9. Let $A \Rightarrow B$ be a fuzzy decision implication and $T \in L^{C \cup D}$. We have $\|A \Rightarrow B\|_{T}=\bigvee\{c \in$ $\left.L \mid\|A \Rightarrow c \otimes B\|_{T}=1\right\}$.

Proof. Since $L$ is a complete MV-algebra, thus is a complete residuated lattice. Similar to Theorem 1 (iii) of Ref. 2, we can prove the above result.

Definition 14. ${ }^{24}$ Let $\mathscr{T}=\left\{T_{1}, T_{2}, \cdots, T_{n}\right\}$. The degree to which $A \Rightarrow B$ holds in $\mathscr{T}$ is defined by $\|A \Rightarrow B\|_{\mathscr{T}}=\bigwedge\left\{\|A \Rightarrow B\|_{T} \mid T \in \mathscr{T}\right\}$.

By Definition 5.4, the following result is obvious.

Theorem 10. For any $\beta \in L \backslash\{0\}$, let $\mathscr{T}=\{T \in$ $\left.L^{C \cup D} \mid R\left(T_{0}, T\right) \geqslant \beta\right\}$. Then $\|A \Rightarrow B\|_{\mathscr{T}}=\| \square_{\beta}(A \Rightarrow$ B) $\|_{T_{0}}$.

Let $X$ be an L-fuzzy set of fuzzy decision implications, that is, $X$ is an L-fuzzy theory. Following the work of Pavelka ${ }^{18}$ by combining with Definition 5.2, the set of models of $X$ can be defined as follows: $\operatorname{Mod}(X)=\left\{T \in L^{C \cup D} \mid\right.$ for each $A \Rightarrow$ $\left.B, X(A \Rightarrow B) \leqslant\|A \Rightarrow B\|_{T}\right\}$. Then the degree to which $A \Rightarrow B$ semantically follows from $X$ is defined by $\mathfrak{C}(X)(A)=\bigwedge_{T \in \operatorname{Mod}(X)}\|A \Rightarrow B\|_{T}$. By Definition 5.5 , we have

$$
\mathfrak{C}(X)(A)=\|A \Rightarrow B\|_{M o d(X)}
$$


Similar to Theorem 5.1, the following result can be obtained.

Theorem 11. Let $A \Rightarrow B$ be a fuzzy decision implication and $X$ an L-fuzzy set of fuzzy decision implications. We have

$$
\mathfrak{C}(X)(A)=\bigvee\left\{c \in L \mid\|A \Rightarrow c \otimes B\|_{\operatorname{Mod}(X)}=1\right\} .
$$

\section{6. conclusion}

This paper focused on the graded semantics of propositional fuzzy modal logic with evaluated syntax based on MV-algebras, and presented its some basic properties. On this basis, we discuss the semantical properties of fuzzy decision implications in propositional fuzzy modal logic with evaluated syntax. It is worth noting that our method is different from that of Ref. 1-5, 20 and Ref. 24, where fuzzy implications or fuzzy decision implications are considered by taking as a whole in fuzzy attribute logic, instead of considering the fuzzy decision implication $A \Rightarrow B$ as a compound formula from the formulas $A \in L^{C}$ and $B \in L^{D}$.

Further, we need to develop the logical calculus of propositional fuzzy modal logic with evaluated syntax corresponding to the established semantics, and build the corresponding proof theory. Meanwhile, we will consider the syntactical characteristics of fuzzy decision implications, and prove the corresponding theorems about the soundness and completeness.

\section{Acknowledgments}

We would like to thank Professor I. Perfilieva and the anonymous reviewers for their very careful, conscientious and helpful comments, all these comments are very helpful for us to improve the quality of the manuscript.

The work was partially supported by the National Natural Science Foundation of China (Grant No. 61100046, 61305074, 61175055) and the application fundamental research plan project of Sichuan
Province(Grant No. 2011JY0092) and the Fundamental Research Funds for the Central Universities of China (Grant No.2682014ZT28).

\section{References}

1. R. Bělohlávek, M. Chlupová and V. Vychodil, Implications from data with fuzzy attributes, in Proceedings of AISTA 2004, Kirchberg, Luxembourg, November 2004, pp. 5.

2. R. Bělohlávek, V. Vychodil, Fuzzy attribute logic: syntactic entailment and completeness, in: JCIS 2005, 8th Joint Conference on Information Sciences, Salt Lake City, Utah USA, 2005, 78-81.

3. R. Bělohlávek, V. Vychodil, Reducing attribute implications from data tables with fuzzy attributes to tables with binary attributes, in: JCIS 2005, 8th Joint Conference on Information Sciences, 2005, 82-85.

4. R. Bělohlávek, V. Vychodil, Attribute implications in a fuzzy setting, in: ICFCA 2006, 2006, 45-60.

5. R. Bělohlávek, V. Vychodil, Pavelka-style fuzzy logic for attribute implications, in: JCIS 2006, 2006.

6. F. Bou, F. Esteva, Exploring a syntactic notion of modal many-valued logics, Mathware \& Soft computing, 15(2008) 175-181.

7. F. Bou, L. Godo, F. Esteva, R.O. Rodriguez, On the minimum many-valued modal logic over a finite residuated lattice, Journal of Logic and Computation, 5(2011) 739-790.

8. C. C. Chang, Algebraic analysis of many-valued logics, Transactions of the American Mathematical Society, 88(1958) 476-490.

9. R. L. O. Cignoli, I.M.L. D’Ottaviano, D. Mundici, Algebraic foundations of many-valued reasoning, Kluwer, 2000.

10. M. C. Fitting, Many-valued modal logics, Fundamenta Informaticae, 15(1992) 235-254.

11. M. C. Fitting, Many-valued modal logics II, Fundamenta Informaticae, 17(1992) 55-73.

12. P. Hájek, On fuzzy modal logics S5(C), Fuzzy Sets Syst. 161(2010) 2389-2396.

13. A.M. Mironov, Fuzzy modal logics, Journal of Mathematical Sciences, 128(2005) 3461-3483.

14. V. Novák,I. Perfilieva, J. Močkoř, Mathematical principles of fuzzy logic, Kluwer Academic Publishers, Boston, 1999.

15. V. Novák, Which logic is the real fuzzy logic?, Fuzzy Sets Syst. 157(2006) 635-641.

16. V. Novák, Fuzzy logic with countable evaluated syntax revisited, Fuzzy Sets Syst. 158(2007) 929-936.

17. V. Novák, Reasoning about methematical fuzzy logic and its future, Fuzzy Sets Syst. 192(2012) 25-44.

18. J. Pavelka, On fuzzy logic I: Many-valued rules of inference, II: Enriched residuated lattices and semantics 
of propositional calculi, III: Semantical Conpleteness of some many-valued propositional calculi. Zeitschr F Math Logik Und Grundlagend Math, 25(1979) 45-52; 119-134; 447-464.

19. S. Pollandt, Fuzzy Begriffe: Formale Begriffsanalyse von unscharfen Daten, Springer-Verlag, BerlinHeidelberg, 1997.

20. K.S. Qu, Y.H. Zhai, J.Y. Liang, M. Chen, Study of decision implications based on formal concept analysis, International Journal of General Systems, 36(2007) $147 \mathrm{C}-156$
21. G.J. Wang, MV-algebras, BL-algebras, $R_{0}$-algebras, and multiple-valued logic, Fuzzy Systems and Mathematics, 16(2002) 1-15.(in Chinese)

22. Y. Xu, Lattice implication algebras, J. Southwest Jiaotong Univ. 28(1993)20-27.(in Chinese)

23. Y. Xu, D. Ruan, K.Y. Qin, J. Liu, Lattice-Valued Logic-An Alternative Approach to Treat Fuzziness and Incomparability, Springer-Verlag Berlin Heidelberg New York Press, 2003.

24. Y.H. Zhai, D.Y. Li, K.S. Qu, Fuzzy decision implications, Knowledge Based Systems, 37(2013) 230-236. 